\title{
Correction
}

\section{Correction: Kamikubo et al., Functional Cooperation of Metabotropic Adenosine and Glutamate Receptors Regulates Postsynaptic Plasticity in the Cerebellum}

In the article "Functional Cooperation of Metabotropic Adenosine and Glutamate Receptors Regulates Postsynaptic Plasticity in the Cerebellum” by Yuji Kamikubo, Takeshi Shimomura, Yosuke Fujita, Toshihide Tabata, Taku Kashiyama, Takashi Sakurai, Kenkichi Fukurotani, and Masanobu Kano, which appeared on pages 18661-18671 of the November 20, 2013 issue, the immunoblot columns in Figure 1, $A$ and $B$, are labeled with the wrong names of antibodies. The description of these data in the corresponding parts of the text (Fig. 1 legend, Results, and Discussion) is correct. The interpretation and conclusions of the study are not affected because this corrigendum only corrects the labeling of the data in the figure. The corrected Figure 1 is shown below and corrected in the online version.

A

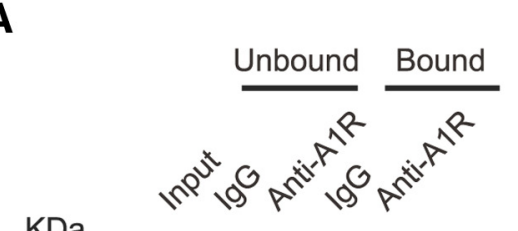

$\mathrm{KDa}$

$--\infty \mathrm{mGluR1}$

150

100 -

75 -

50 -

37 -

C
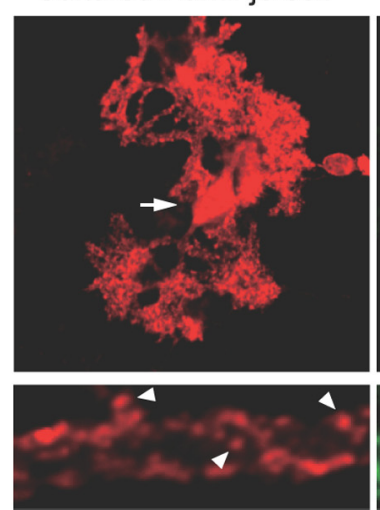

mGluR1
B $\mathrm{KDa}$<smiles>CCOCCOCCOCCOCC</smiles>

250 -

150 -

100 -

75 -

50 -

37 -
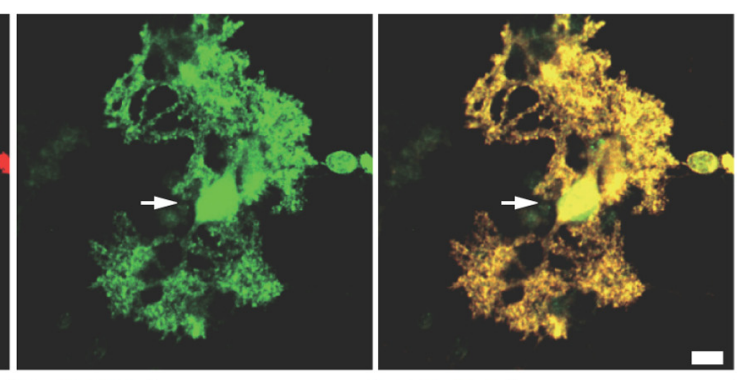

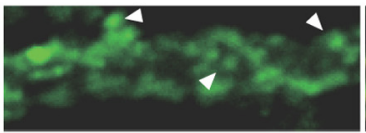

A1R

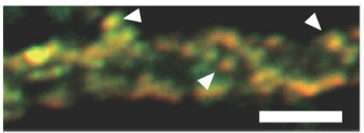

Overlay

Figure 1. 